\title{
Designing Mediated Combat Play
}

\author{
Florian 'Floyd' Mueller ${ }^{1,2}$ Martin Gibbs $^{2}$ Frank Vetere $^{2}$ Stefan Agamanolis $^{3}$ Darren Edge $^{4}$ \\ ${ }^{1}$ Exertion Games Lab \\ RMIT University \\ ${ }^{2}$ Computing \& \\ Information Systems \\ ${ }^{3}$ Akron Children's \\ ${ }^{4}$ Microsoft Research \\ Melbourne, Australia \\ University of Melbourne, AUS \\ Hospital \\ Beijing \\ floyd@exertiongameslab.org, \{martin.gibbs, f.vetere\}@unimelb.edu.au, stefan@agamanolis.com, \\ darren.edge@microsoft.com
}

\begin{abstract}
Supporting physical exertion is a growing trend in digital technology design. However, most experiences focus on bodily actions in which participants act independently of each other. In contrast, we focus on virtual body-to-body interactions between multiple participants, inspired by combat-oriented sports such as boxing that highlight the need to act while avoiding reciprocal bodily action. Mediating such body-to-body interactions with technology is challenging, particularly when participants are not colocated. Prior systems have often involved a mixture of novel physical interfaces and interactions through virtual avatars. This paper charts a design space for virtual combat play experiences and offers a set of design dimensions and recommendations for future systems. We draw on our experiences of designing and evaluating Remote Impact - a boxing-style exertion game involving aggressive bodily interaction with a large force-sensing projection surface. By expanding our knowledge of mediated exertion with an understanding of combat interactions we extend the social experience space of exertion play.
\end{abstract}

\section{Author Keywords}

Exertion game; exertion interface; whole-body interaction; exergame; sport; combat; distributed interaction

\section{ACM Classification Keywords}

H.5.2 Information Interfaces and Presentation: User Interfaces

\section{INTRODUCTION}

There has been a growing interest in digital technology that supports bodily actions that result in physical exertion [18]. Such exertion interactions benefit from and contribute to social play [11, 18]. Popular gaming consoles such as Nintendo's Wii, Microsoft's Xbox Kinect and Sony's

Permission to make digital or hard copies of all or part of this work for personal or classroom use is granted without fee provided that copies are not made or distributed for profit or commercial advantage and that copies bear this notice and the full citation on the first page. Copyrights for components of this work owned by others than the author(s) must be honored. Abstracting with credit is permitted. To copy otherwise, or republish, to post on servers or to redistribute to lists, requires prior specific permission and/or a fee. Request permissions from Permissions@acm.org.

TEI'14, February 16 - 19 2014, Munich, Germany

Copyright is held by the owner/author(s). Publication rights licensed to $\mathrm{ACM}$.

ACM 978-1-4503-2635-3/14/02 \$15.00.

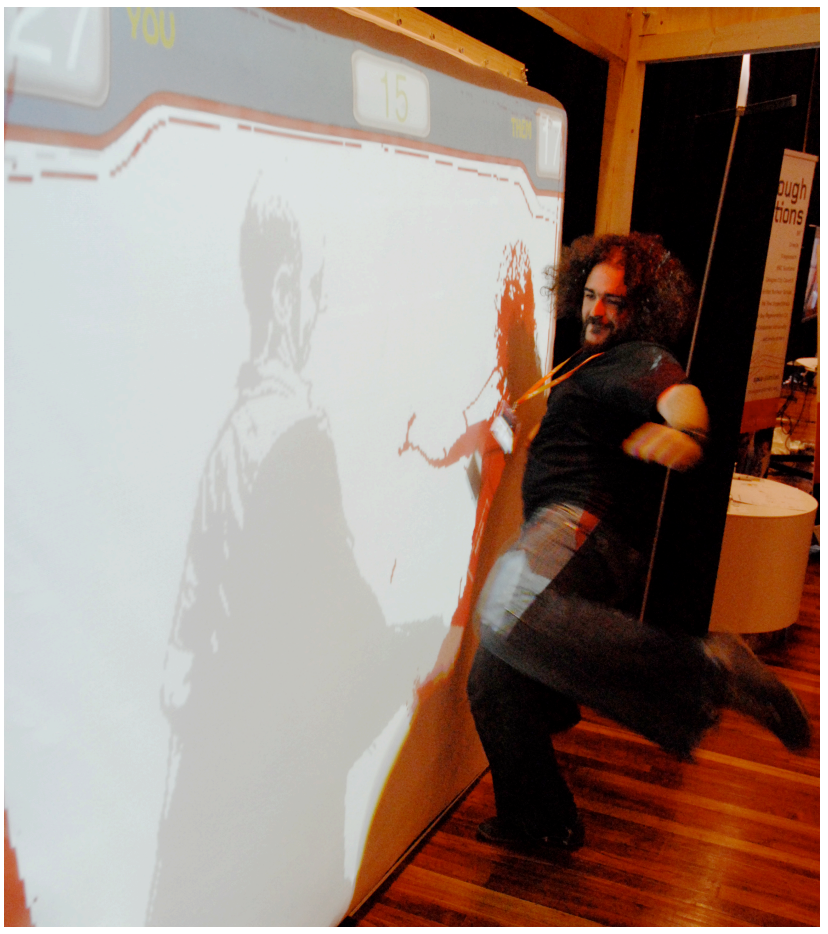

Figure 1. Remote Impact player striking

Playstation Move increasingly support such multi-player co-located exertion play, yet many games are not utilizing mediated body-to-body interactions. By body-to-body interactions we mean interactions in which bodies act on and react to each other, going beyond touch interactions (as often explored in HCI in an arts context, for example see [9]). Many current commercial games support mostly independent activities, where players take turns or play in parallel [26] (e.g., games oriented on aerobics, golf, jogging, yoga). These activities contrast with traditional sports, which often feature extensive body-to-body interactions, as in boxing, American football and rugby. If console games have attempted to lean on these body-tobody interactions (for example the Madden series), they restrict bodily interactions to avatars, and fall short when it comes to body-to-body interactions between players. The limitations of sensing and actuating technologies to support such body-to-body interactions may be part of the reason: for example, the underlying sensor technologies in the 
Wiimotes, Kinect cameras and Move controllers exacerbate the separation of bodies because they require players to stand apart, thus limiting opportunities for body-to-body interaction. In contrast, in this paper, we focus on bodily combat experiences mediated through technology.

Mediating body-to-body interaction becomes even more challenging when participants are geographically separated, out of direct contact of any physical gestures made by one another. Our work offers ideas and recommendations on how such interactions can be conveyed over a distance.

The main contribution of this paper is an understanding of the design of technology-mediated combat-style experiences. We scaffold this understanding in two mutually supportive ways. Firstly, we explore current knowledge about bodily interactions that are mediated through computer technology. We then report on seven core findings arising from our study of Remote Impact, a boxing-like exertion game designed to support body-tobody combat-style experiences between distributed participants [15]. We end with recommendations about how careful mapping between physical bodies and their virtual world representations can be used to design engaging combat style experiences. We acknowledge that as we are inspired by combat sports such as boxing, our focus is on bodily interactions in a competitive context (in contrast to the collaborative lineout lifting in rugby for example). However, we believe our work can be a valuable starting point for broader investigations into bodily interactions including larger team involvement.

Our work adds insights about combat-style interactions to the understanding of exertion systems and offers a structured approach towards expanding the social experience space for participants. In turn, this will give players a richer set of opportunities to reap the benefits of exertion. The results can be applied to the design of exertion games that aim to support bodily interactions between players. In particular, it can inform the design of future games and hardware add-ons for platforms such as Wii, Move and Kinect. Our work can be used to inform the design of augmented gym equipment that aims to expand the social experience space for exercisers. It might also be beneficial to the design of mobile apps that support exertion activities while considering mediated social support. Lastly, our work can be applied to the design of sports equipment that augments existing bodily interactions, such as interactive protective guards for Taekwondo [4]. In the next section, we identify the need for a more formal understanding of mediated combat-style experiences.

\section{MEDIATING BODILY INTERACTIONS}

Previous research has touched upon how interactive technology can support participants interacting with each other on a bodily level. Some of these explorations support social exertion activities, while other systems have emerged that focus on mediated bodily interactions.
Ludvigsen et al. support our idea of a "bodily interaction" continuum by articulating that there is a range of how bodies can relate to one another in sports; furthermore the authors argue that it is important for interaction designers to consider the entire range of bodily interaction possibilities [13]. In response, they introduce an interactive system for professional handball players' training sessions, in which the players have to anticipate their partner's movements in order to press illuminated buttons before their partner does. The authors call this idea of athletes reacting to other athletes' movements "kinesthetic empathy" [5] and propose that interaction designers regard it as an opportunity for design. We see our work on combat-style interactions as a subset of the larger kinesthetic empathy interaction space.

Chi et al. were the first to introduce the HCI community to combat-style interactions by presenting an augmented wearable chest protector for martial arts competitors [4]. This work highlights the potential of technology to enhance the combat-style experience. Boxing games that sense body movement, such as the Wii boxing game [21] or the Kinect boxing games $[25,27]$ are similar to our prototype in that they draw on boxing actions and augment the associated combat-style experiences with digital game elements. Research suggests that players can find such non-contact combat-style games engaging [22], and our Remote Impact work aims to push engagement levels even further by stimulating the visceral feelings that lead both to and from the experience of body-to-body interactions.

In sum, prior work suggests that bodily interactions where participants act on and react to each other's bodily actions are not often explored when it comes to interactive technology, and that this is a missed opportunity to expand the exertion experience space. Missing this opportunity means that exertion games will probably continue to focus on offering individual exercises, rather than facilitating rich social bodily experiences that people know from traditional sports. Our work aims to address this gap by providing an initial understanding for a subset of combat-style interactions based on striking. By doing so, we aim to offer a starting point towards building knowledge about how interactive technology can contribute to this bodily interaction space.

We have previously discussed the motivation for Remote Impact [17] and briefly described its technical implementation in two short papers $[14,16]$. We now extend this work by reporting fully on the design outcomes and a detailed understanding of technologically oriented tactics for body-to-body interactions. We begin by reviewing Remote Impact and then describe a study we conducted and discuss the findings of both.

\section{REMOTE IMPACT}

Players of Remote Impact (Fig. 1) face a playing area on which the shadow of the remote person is projected. Their own shadow is also displayed in a different shade of grey. The interaction surface, made out of a combination of 
mattresses, ripstop and lingerie fabric, spans an area of 2.10 x 2.50 meters. The shadows appear to be created by a light source behind the players, i.e., if the players step closer to the camera behind them, their shadows increase in size. The players can also talk to and hear each other through an audio connection between the locations. Although Remote Impact can support up to two players on each end, we focus on one-on-one combat play as a starting point.

Once the game starts, both players try to strike each other's shadow. They can target any area of their opponent's shadow, and strike with their palms, fists, feet or entire body (Fig. 2). An impact on the remote person's shadow area is considered a successful hit. The impact of the user's body onto the surface is measured by detecting the deformation of the surface area. The higher the intensity of the hit, the higher the points scored. If a hit is placed within the shadow area of the remote person, a visual indicator is displayed on the impact spot and a sound effect is played to indicate to both players that a successful hit occurred. If the player missed, a different visual appears, indicating that no points were added to the score. The player with the most points within the time limit wins the game.

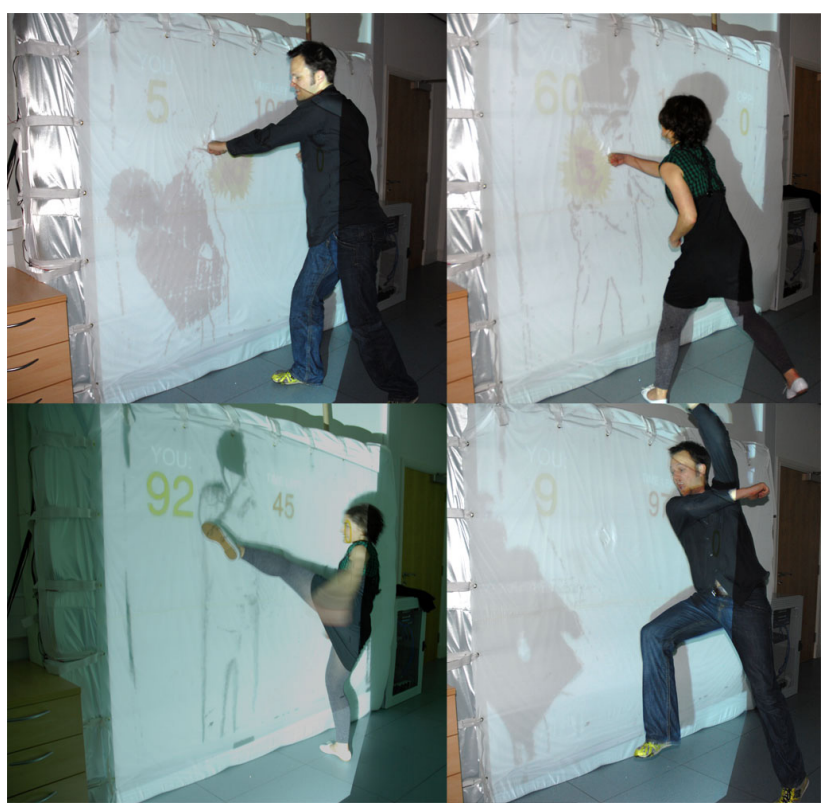

Figure 2. Combat actions with Remote Impact

\section{STUDY}

We exhibited Remote Impact at two public events staged in our lab, as well as at two conferences in two different countries. We also conducted a formal study to understand what it was like for participants to engage with Remote Impact. For this study, we recruited 20 participants (14 female) of average age 31, asking for no prior combat experience, as our game was not targeting combat experts (unlike Chi et al.'s system [2]). We had asked the volunteers to bring a partner along, so we had 10 pairs that were either friends or siblings. The two Remote Impact stations were placed in separate spaces of the same building. Each study round took one hour. All participants arrived after (desk-based) work, meaning they did not engage in an exertion activity beforehand. We believe the demographic for mediated combat games is yet to emerge, and hence we chose not to be restrictive in the selection of participants.

We used video to capture bodily gameplay as successfully demonstrated previously by researchers concerned with the moving body [12]. Participants were also interviewed together after the game about their experience, where we took notes and the interviews were transcribed. We used an iterative coding process to identify key analytic categories. We then placed these categories in a spatial affinity diagram in order to derive dominant themes. The aim was to draw conclusions based on a systematically developed and conceptually coherent understanding of the data.

\section{ENGAGING WITH REMOTE IMPACT}

Overall, the participants said they thoroughly enjoyed Remote Impact. Participants exerted themselves with forceful strikes towards their partner's shadow and ducked out of the way when strikes were coming towards them. They applauded the system for providing them with an engaging exertion experience: "This is great!", "This is so much fun!" and "I am more exhausted than I was when I played squash." We now describe the specific strategies participants used and the practices that arose when engaging with Remote Impact.

\section{Finding 1: Full body engagement}

Players exerted themselves extensively by using their entire body to strike the interactive surface. Participants predominantly used their fists, but everyone also used their feet to kick. Some players also threw their entire bodies against the surface. Participants appeared to switch from punches to kicks when their arms got tired, which allowed them to continue exerting themselves.

\section{Finding 2: Force intensity as challenge}

Participants exhibited a great deal of brute force during their play. Most hits appeared to use maximum force, and players seemed to enjoy the ability to apply extreme intensity in their physical actions. A major challenge for participants appeared to be hitting with such intensity: "It's a lot harder than you think." The result of this challenge arose in the form of exhaustion: "[Exhaustion noise] I need some water." Seeing force intensity as a challenge was further exemplified by two friends who engaged in a mini competition after the study about examining who can hit the interactive surface harder: they took turns in hitting the surface with one strike as hard as they could.

\section{Finding 3: Force as stress relief}

The ability to apply intense force also functioned as stress relief, which participants applauded: "It's a great stress relief." In the interviews, participants pointed out the contrast between the types of force interactions the Remote Impact system facilitates and the types of interactions in their normal environments such as offices, where brute force is discouraged. In particular, they highlighted that 
technology usually needs to be handled with care. In contrast, Remote Impact gives participants an opportunity to apply stress-relieving force in a social context.

\section{Finding 4: Bodily communication and self-expression}

Participants used their bodies and in particular the associated shadow representations (with their varying sizes) to communicate to their partner and to express themselves. For example, one player made a victory sign with her hands to communicate the enjoyment of a win to their partner. Another participant did a hang-ten sign: upon seeing her gesture projected onto the surface, she then moved her hands closer to her head to explore the horns she created, similar to the way people play with their hands to create shadows. So the shadows facilitated further bodily play for participants, supporting rich forms of self-expression.

\section{Finding 5: Strategic play}

Some participants also noted that after playing for a while, they changed their strategy from more "traditional" boxing behavior, where strikes were interspersed with ducking moves, to mostly rapid strikes applied in quick succession. This more offensive-oriented strategy did not necessarily secure a win more easily (as this enabled the other player to also apply more strikes), but it resulted in a higher score overall. Participants appeared to enjoy the opportunity to engage with this offensive strategy, as it allowed them to execute more hits: "It just feels great, hitting this mattress." The realization that a more offensive striking strategy results in more opportunities to hit the interactive surface encouraged players to hit as fast and as hard as they could. This reduction in strategic play resulted in a reduced sense of playing together, described as "more like playing against a computer."

\section{Finding 6: New bodily experiences due to reduction of physical risk}

Another theme that emerged was the reduced physical risk participants perceived compared with the risks of traditional combat-style experiences: "It's good because you can't get hit." Players said that there was little incentive for them to engage in defensive actions: getting hit meant that the other player scored points, but there were no other drawbacks such as pain. Participants compared Remote Impact with boxing, but stressed that the difference in perceived physical risk made Remote Impact a different kind of experience: "This is not boxing, the sport."

\section{Finding 7: Playing with tracking limitations}

We also observed participants incorporating the limitations of the camera-based tracking system into their play strategies. Players realized that the conical capture area of the camera spanned a large zone where most movement occurred; however, the camera did not track the entire room. Participants used this borderline between these two areas as part of their play strategy. For example, a pair of players realized that if they lie with their backs on the floor and kick the interactive surface with their feet, approximately half of their bodies were not captured, reducing the area on which they could be hit. Another player ran from outside the capture area into the capture area to use this sudden appearance to hit her partner by surprise, accompanied by lots of laughter.

\section{DESIGNING COMBAT-STYLE EXPERIENCES}

We now discuss ways of designing combat-style experiences based on our craft knowledge of creating Remote Impact. Our experiences of exhibiting Remote Impact and the data from the study further helped us refine this knowledge. We identified that mappings are an important consideration when designing combat-style experiences, and by reflecting on our experiences, we are able to articulate a set of key mappings. By mapping we mean the mapping between the physical bodies and their virtual world representations in the form of the players' avatars (these representations do not have to be visual, however, the shadows in Remote Impact are visual and for simplicity we call them avatars). We believe that these mappings can help form an important initial understanding of how to design for combat-style experiences.

We explain the mappings through Remote Impact, but we also refer to three other types of combat-style games in order to demonstrate the wider applicability of our thinking as well as highlight underexplored areas of the design space (Fig. 3): Wii Boxing [21] is a commercial boxing game with virtual boxers controlled by players using Wiimotes, similar to Kinect Sports Boxing [27] that uses the Kinect. Propinquity [29] is a research game that facilitates combatstyle movements through encouraging players to hold their hands over their partner's sensors attached to various limbs on their bodies, triggered by proximity (touch is not allowed according to the rules). Supported by music, the result is a capoeira-like dance players engage in. This game along with the similar JS Joust [30] engages combat-style play. Although Propinquity does not focus on mediated interactions, we include it to suggest the applicability of the mappings to even these non-avatar types of combat games.

\section{Mapping 1: Motion Mapping}

We identified four key ways designers can map the extent of movement from the physical to the virtual world.

Literal one-to-one mapping: Remote Impact sensed participants' entire body movements and mapped them to a virtual avatar (within limits, see below). As such, the mapping is a literal one, almost one-to-one, where, for example, a physical strike was represented as a virtual strike with roughly the same speed, shape and velocity. By supporting the full body (F1), players were able to use their hands, feet, the torso etc., which allowed them to engage in a rich set of tactics. Kinect Sports Boxing also supports a literal one-to-one mapping, however, Wii Boxing does not, which we explain next.

Partial one-to-one mapping: Wii boxing [21] supports only a subset of bodily movements. Wii boxing senses participants' hands only through the handheld controllers and ignores movements of the rest of the body. As the mapping is a one-to-one mapping, the player's hand 
movements control the avatars' boxing gloves, but the avatar's torso does not move along with the player moving. As such, players do not have the opportunity to use their torso for bodily tactics. We note that although Remote Impact supports movement to a larger extent than Wii boxing, it did not consider the full extent of participants' movements, as the example of the participants lying on the floor suggested. Those participants played with the borderline between what is tracked and what is not (F7), supporting the idea of seeing tracking limitations as opportunities for design [1].

Complexity-reducing mapping: Designers can also choose to reduce the complexity of movements, for example, full body movement can be tracked, but then turned into more simplified representations in the virtual world. Nijhar et al. [20] point out that considering this mapping can be particularly useful for engaging players who are motivated to play to "relax", rather than to "achieve". Although Remote Impact predominantly engages literal mapping, we can also see how complexity-reduced mapping can play a role. In Remote Impact, the full body was tracked, however, the virtual representation was not a copy of the body, but a simplified version in the form of a shadow, reducing complexity. For example, players could see a strike coming, but it was difficult to identify which way a fist was turned, simplifying tactical decisions. Furthermore, Remote Impact allowed participants to play with this body mapping, supporting their desires for bodily communication and selfexpression (F4). Propinquity's motion mapping is closest to complexity-reducing mapping: the bodily proximity players aim for is reduced to a scoring mechanism where proximity within a range (such as $1 \mathrm{~cm}$ to $15 \mathrm{~cm}$ ) scores a point.

Complexity-increasing mapping: Designers can also choose to utilize the mapping in order to add complexity to movements. Such an approach is often deployed in buttonpress fighting games such as the special moves dominant in the Street Fighter game series [28]. There, only a small subset of movements are sensed (finger presses), but mapped to highly complex movements executed by virtual characters. In a modified version of Remote Impact for example, combinations of strikes could temporarily decouple the player from his/her shadow while it performed a particularly potent special move, well above normal intensity levels, such as proposed by Kick Ass Kung Fu [8]. Sensing only limited movement and adding complexity computationally can support the fantasy experience of players [23]: Street Fighter supports players in experiencing a fantasy where they have excellent combat skills even though in reality they do not.

\section{Mapping 2: Location Mapping}

When mapping movement to a virtual representation, designers need to consider physical disparity. By physical disparity we mean the physical distance between the bodily action and its representation. In Remote Impact, it is the distance between a movement (for example a player raising his/her arm) and the virtual representation of that movement (the shadow on the interactive surface). Designers can choose to support physical disparity to a greater or lesser extent, which affects opportunities for bodily interaction. Furthermore, Remote Impact suggests that designers can mix different physical disparities for different actions, varying them throughout an experience.

Small disparity: In Remote Impact, participants experienced little physical disparity when they were hitting the interactive surface: the distance between their fist or foot and the virtual representation was zero at the point of impact. Small physical disparity supports the proprioceptive sense, as participants' movements "directly" relate to the virtual representation: similar to a touchscreen, the user acts on the virtual representation through direct manipulation, no complex mental mapping between physical movement and virtual representation is required. This probably explains the success of Remote Impact in relieving stress (F3), as there were limited mental demands due to the small physical disparity, allowing participants to focus solely on physical actions. Games that deploy sensors attached to the player's body such as Propinquity usually feature small disparity and benefit from the fact that no complex mental mapping is required.

Large disparity: When Remote Impact players were dodging out of the way, the physical distance between their bodies and the virtual representation was usually around 1-1.5 meters, much larger compared to when striking the surface. Here, players needed to engage a mental mapping process where they had to consider how much they needed to move in order for their virtual shadow representation to avoid the strike. Large physical disparity is often deployed to make the mapping more efficient, e.g., movements of a computer mouse are normally smaller than movements of the associated cursor, reducing the physical effort required. This runs contrary to the premise of many exertion games that aim to encourage and facilitate physical effort. In Remote Impact, increasing the physical disparity meant stepping further away from the interactive surface, and hence closer to the camera. Due to the conical shape of the capture area of the camera, participants' shadows got bigger, so players created larger representations of themselves. This allowed, for example, a small arm movement to turn into a very large virtual swing; participants used this to stimulate their play (F4).

\section{Mapping 3: Force Mapping}

Players of Remote Impact appreciated the opportunity to apply brute force [17] to the interaction surface (F2), which affected the visceral feeling of the experience. Prior work by Berthouze has suggested that this is important, as it can facilitate players' feelings of presence and fantasy [2]. We propose that designers should consider the extent to which the force of movements is sensed and mapped.

Unit force: Designers can choose to ignore the amount of force applied during participants' movements and simply detect if an action was successful or not. This can simplify 
the sensing implementation and also support balancing [19], allowing players of different strengths to play together. In Propinquity the force of movements is not sensed, and in Wii Boxing, the force of movements is mapped discretely to the avatar, so that only once a hit reaches a certain threshold, it results in a harder avatar punch.

Functional force: Probably the most-liked feature of Remote Impact was that harder hits resulted in larger scores. The force of movements was sensed and mapped in Remote Impact, which rewarded participants' physical effort. However, the force was not mediated across the distance, for example, players did not experience a hit through forcefeedback devices. This affected participants' body-to-body interactions: their focus sometimes shifted from force investment as a way to drive bodily interactions to force investment as a way to engage in parallel competitions about determining who can hit the hardest (F2).

\section{Mapping 4: Control Mapping}

Designers need to consider the extent to which bodies have control over other bodies. In mediated environments this translates to the extent bodies have control over avatars and vice versa, but also includes to what extent avatars have control over other avatars. As such, we argue that designers need to consider both the mapping between bodies and avatars and the mapping between avatars. For both kinds, designers have two broad choices:

Unidirectional mapping between body and avatar: Remote Impact, just like Wii Boxing, features a unidirectional mapping between the player's body and the virtual shadow, the player's avatar. If a player moves, the avatar moves. This mapping is unidirectional, as any movement of the avatar does not result in the player moving. If the opponent hits the avatar, the player does not experience this hit in a bodily sense: the only feedback the player receives is the cartoon-style visuals and audio effects (F6). The lack of bodily feedback meant that there were few incentives to engage a mix of different tactics. As a result, some players focused on simple hitting actions, meaning that the unidirectional mapping between body and avatar affected the bodily interactions between participants (F5).

Bidirectional mapping between body and avatar: Alternatively, designers can choose to support a bidirectional mapping between the player's body and his or her avatar. In Remote Impact, if an avatar was struck, the player did not experience this with his or her body. However, we can envision that with advances in forcefeedback technology this could be supported. Regardless of whether this bidirectional mapping is technically feasible, designers should consider any ethical and safety concerns when computers offer bodily feedback, in particular when it can be forceful as in combat-style interactions.

Loose mapping between avatars: Remote Impact featured a loose bodily mapping between avatars: if an avatar was struck, a point was scored, however, the avatar did not experience this bodily, i.e. the avatar did not move backwards as a result of the impact.

Tight mapping between avatars: Wii Boxing features a relatively tight mapping between avatars: a struck avatar experiences the hit bodily, most often in the form of moving backwards. This does not lead to a broken motion mapping (M1), as the avatar's body is not tracked. It should be noted that the design of Remote Impact did not aim to offer a completely loose mapping between avatars. Although avatars did not react to bodily actions, the experience for players hitting their partner's avatar was one where they felt resistance through the mattress surface. Furthermore, the avatar seemingly moved as a result of the input: the surface was squashed, providing the sensation something "gave" and reacted to the physical action in a tangible way. Since the projection also follows the squashed surface, this created the illusion that the avatar being hit was reacting to the physical action. Remote Impact therefore tightened the mapping between avatars by facilitating perceived reactions such as the projection on the malleable surface and offering tactile feedback in addition to the visual and audio effects. Interestingly, by using proximity and wearable sensors Propinquity portrays a tight mapping between avatars even though it does not feature virtual representations in the form of visual avatars.

\begin{tabular}{|c|c|c|c|}
\hline \multicolumn{4}{|c|}{ Motion Mapping } \\
\hline R K & $\mathbf{W}$ & $\mathbf{P}$ & \\
\hline Literal & Partial & $\begin{array}{l}\text { Complexity } \\
\text { reducing }\end{array}$ & $\begin{array}{l}\text { Complexity } \\
\text { increasing }\end{array}$ \\
\hline \multicolumn{4}{|c|}{ Location Mapping } \\
\hline $\mathbf{P}$ & $\mathbf{R}$ & K W & \\
\hline Small & & & Large \\
\hline \multicolumn{4}{|c|}{ Force Mapping } \\
\hline & K W P & $\mathbf{R}$ & \\
\hline Unit & & & Functional \\
\hline \multicolumn{4}{|c|}{ Control Mapping } \\
\hline R K & $\mathbf{W} \mathbf{P}$ & & \\
\hline Uni+loose & Uni + tight & $B i+$ loose & $B i+t i g h t$ \\
\hline $\mathbf{R}_{\text {Impact }}^{\text {Remote }}$ & W ${ }_{\text {Boxing }}^{\text {Wii }}$ & $\mathbf{K} \begin{array}{l}\text { Kinect } \\
\text { Boxing }\end{array}$ & P Propin- \\
\hline
\end{tabular}

Figure 3. Mappings in combat games.

\section{TACTICS FOR DESIGNING COMBAT-STYLE EXPERIENCES}

We now present a set of design tactics we derived from our study of Remote Impact. These are aimed at providing designers with practical guidance as to what extent different mappings can facilitate different experiences. The design mappings present the range of available options for designers in a value-neutral fashion, while the tactics add value to these mappings. 


\section{Mapping 1: Motion Mapping}

Tactic 1.1: Exploit sensing seams as a resource for body-tobody interactions: Remote Impact participants played with the limitations of the sensing technologies to engage with their partner on a bodily level (F7): The use of sensing boundaries - what is sensed, and what is not and the ambiguous area in-between - has previously been promoted as a resource for design by the name of seamful design [3]. Here we advocate exploiting those sensing seams as a design resource to support bodily interactions.

Tactic 1.2: Map the whole body to facilitate rich opportunities for tactics: The Remote Impact study suggests that the ability to use the full body gave players a rich set of opportunities to engage in different types of tactics as part of their bodily experience (F1). The work by Forlizzi [6] reminds us that interactive technology can contribute to the emergence of rich experiences: here we refine this and suggest to designers that mapping the entire body (in contrast to only some body parts) affords rich opportunities for tactical engagement that in turn affords rich bodily interactions with other people, even when mediated. This tactic is useful for designers of sensing systems such as the Kinect as it can help them argue why sensing the entire body is advantageous to sensing only parts of it: interacting with the torso, hands and feet (in contrast to just one controller) is not just more exerting, it also allows for richer tactics to support body-to-body interaction.

\section{Mapping 2: Location Mapping}

Tactic 2.1: Exploit different physical disparities: Remote Impact players engaged with the varying physical disparity, which contributed positively to their exertion experience (F1, F4). So far, most system designs focus on either large or small physical disparities, here we argue that varying physical disparity within the same experience can have benefits: in Remote Impact, players engaged with the physical disparities afforded by the system through moving close and away from the interaction surface, which contributed to their exertion. Designers of new interactive hardware, such as touch screens, depth-sensing cameras and wearable sensors are encouraged by this tactic to combine their sensors' features in order to support a variety of physical disparities. The result will be support for bodily interactions across a larger interaction space, contributing body-to-body considerations to the emerging trend on proxemics interactions [7].

\section{Mapping 3: Force Mapping}

Tactic 3.1: Consider force to support bodily interaction: Participants applauded the opportunity to exhibit force as part of their bodily interactions (F2, F3). Force is an important element of bodily interactions; sportspeople know about the delicate borderline between when a forceful action is understood as dedicated play and when it becomes an unnecessary hostile attack. Managing force in bodily interactions is part of the social interaction players engage in, contributing a part of the "invisible" contract between players, marking the "magic circle" of play [23]. Designers need to be aware that exhibiting force can be part of engaging body-to-body interactions (F2), but can also be a cause for leaving the magic circle.

Tactic 3.2: Do not apply a limit to the extent of force in the bodily interaction: Remote Impact participants applauded the opportunity to play with the extent of force they could apply, some even turning it into a competition about who can strike the hardest (F2). The hardware and software did not apply any constraint on the extent of force that could be measured and mapped; in fact, pilot studies made us change the physical sensor system in order to allow for measuring higher force values. We recommend that designers ensure that even the most forceful interactions are sensed and mapped meaningfully, in contrast to maxing out at arbitrary values such as 255 .

\section{Mapping 4: Control Mapping}

Tactic 4.1: Alter physical risk to facilitate novel experiences: Remote Impact players appreciated that the system enabled a new type of experience when compared to traditional boxing as a result of the altered risk (F6). We therefore recommend that designers consider altering physical risk (or the perception thereof) as a means to facilitate novel experiences. Previous work highlights the importance of physical risk in bodily interactions [10], here we extend this work and argue that designers who are working on the body-avatar relationship have unique opportunities to alter physical risk due to the mediation involved that can result in novel experiences. For example, technologies such as interactive protective gear [4] could sense bodily interactions and change the perception of any associated risks based on the level of physical danger they detect. This tactic might inspire designers who so far mainly worked on recreating physical experiences in the virtual world (such as in virtual reality research) to regard any arising physical risk not as a problem to address, but rather as an opportunity to facilitate novel experiences.

\section{CONCLUSIONS}

Our work highlights the emerging area of mediated bodyto-body interactions. We presented research on Remote Impact, an exertion game designed to support combat-style experiences. By analyzing participants' experiences, we identified the characteristics that made playing Remote Impact engaging. Furthermore, the research provided an understanding of the design mappings that influence mediated combat play, extending our knowledge of mediated bodily interactions. Establishing such an understanding now is important, as novel sensing systems are emerging that can be easily extended to support combat play (for example see [24]). Our work therefore presents a timely understanding that serves as foundation for the evolving field of body-to-body interactions. In this paper we focused on the benefits of bodily interactions, however we also wish to highlight the potential dangers that can occur with technology that supports extreme bodily interactions. For example, we know from combat sports that physical injury can occur. The computer augmentation 
might increase the danger that comes with these interactions or might desensitize participants to the danger involved if not designed sensibly. Identifying the opportunities and shortcomings when it comes to the dangers of mediating bodily interactions is an important avenue for future work.

We expect our work on combat-style experiences will assist designers in supporting rich bodily interactions that are engaging and entertaining. We hope that in consequence, participants increase their participation in these bodily interactions and, as a result, that more people profit from the many benefits of engaging in exertion activities.

\section{ACKNOWLEDGEMENTS}

Thanks to Matthew Karau, Costas Bissas, Cindy Jeffers, Elena Corchero, Richard Wilson, Andrea Taylor, Paula Nichols, Angus Aitken, Tomoko Hayashi, Hilary Grant, the Interaction Design Group, the Australian Research Council as well as Distance Lab in Scotland where Remote Impact was developed and the University of Melbourne and RMIT University where it was analyzed.

\section{REFERENCES}

1. Benford, S., Schnädelbach, H., Koleva, B., Anastasi, R., Greenhalgh, C., Rodden, T., Green, J., Ghali, A., Pridmore, T. and Gaver, B. Expected, sensed, and desired: A framework for designing sensing-based interaction. TOCHI, 12 (1). (2005), 3-30.

2. Bianchi-Berthouze, N. Understanding the role of body movement in player engagement. Human-Computer Interaction, 28 (1). (2013), 40-75.

3. Chalmers, M., Bell, M., Brown, B., Hall, M., Sherwood, S. and Tennent, P., Gaming on the edge: using seams in ubicomp games. $A C E^{\prime} 05$, ACM, 306-309.

4. Chi, E.H., Song, J. and Corbin, G., "Killer App" of wearable computing: wireless force sensing body protectors for martial arts. UIST'04, ACM.

5. Fogtmann, M.H., Kinesthetic Empathy Interaction Exploring the Possibilities of Psychomotor Abilities in Interaction Design. In Workshop on Physicality, 2-3 September 2007, Lancaster University, UK, 2007.

6. Forlizzi, J.L. Designing for Experience: An Approach to Human-Centered Design, Thesis, 1997.

7. Greenberg, S., Marquardt, N., Ballendat, T., DiazMarino, R. and Wang, M. Proxemic interactions: the new ubicomp? interactions, 18 (1). (2011), 42-50.

8. Hämäläinen, P., Ilmonen, T., Höysniemi, J., Lindholm, M. and Nykänen, A., Martial arts in artificial reality. CHI'05, ACM New York, NY, USA, 781-790.

9. Hobye, M. and Lowgren, J. Touching a stranger: Designing for engaging experience in embodied interaction. Journal of Design, 5 (3). (2011), 31-48.

10. Klemmer, S. and Hartmann, B., How Bodies Matter: Five Themes for Interaction Design. DIS'06, 140-149.

11. Lindley, S.E., Le Couteur, J. and Berthouze, N.L., Stirring up experience through movement in game play: effects on engagement and social behaviour. $\mathrm{CHI}^{\prime} 08$, ACM, 511-514.
12. Loke, L., Larssen, A., Robertson, T. and Edwards, J. Understanding movement for interaction design: frameworks and approaches. Personal and Ubiquitous Computing, 11 (8). (2007), 691-701.

13. Ludvigsen, M., Fogtmann, M. and Gronbek, K., TacTowers: an interactive training equipment for elite athletes. DIS'10, ACM, 412-415.

14. Mueller, F., Agamanolis, S., Gibbs, M. \& Vetere, F., Brute Force Interface: Leveraging Intense Physical Exertion in Whole Body Interactions. Workshop on Whole-Body Interactions. CHI'09, Ext. Abstracts, 1-4.

15. Mueller, F., Agamanolis, S., Gibbs, M. and Vetere, F., Remote Impact: Shadowboxing over a Distance. In CHI'09, Extended Abstracts, ACM, 3531-3532.

16. Mueller, F., Agamanolis, S., Gibbs, M. and Vetere, F., Remote Impact: Shadowboxing over a Distance. CHI'08, Extended Abstracts, ACM, 2291-2296.

17. Mueller, F., Agamanolis, S., Vetere, F. and Gibbs, M., Brute force interactions: leveraging intense physical actions in gaming. $\mathrm{OzCHI}$ '09, ACM, 57-64.

18. Mueller, F., Edge, D., Vetere, F., Gibbs, M.R., Agamanolis, S., Bongers, B. and Sheridan, J.G., Designing Sports: A Framework for Exertion Games. CHI'11, ACM, 2651-2660.

19. Mueller, F., Vetere, F., Gibbs, M., Edge, D., Agamanolis, S., Sheridan, J. and Heer, J., Balancing exertion experiences. CHI'12, ACM, 1853-1862.

20. Nijhar, J., Bianchi-Berthouze, N. and Boguslawski, G. Does Movement Recognition Precision Affect the Player Experience in Exertion Games? Intelligent Tech. for Interactive Entertainment, Springer, 2012, 73-82.

21. Nintendo. Wii Sports, http://wii.nintendo.com/software_wii_sports.html.

22. Pasch, M., Berthouze, N., van Dijk, E.M.A.G. and Nijholt, A., Motivations, Strategies, and Movement Patterns of Video Gamers Playing Nintendo Wii Boxing. In Facial and Bodily Expressions for Control and Adaptation of Games, 2008, 27-33.

23. Salen, K. and Zimmerman, E. Rules of Play: Game Design Fundamentals. MIT Press, USA, 2003.

24. Sato, M., Poupyrev, I. and Harrison, C., Touche: enhancing touch interaction on humans, screens, liquids, and everyday objects. CHI'12, ACM, 483-492.

25. Ubisoft. Fighters Uncaged, http://fightersuncaged.uk.ubi.com/.

26. Vossen, D.P. The Nature and Classification of Games. Avante, 10 (1). (2004), 53-68.

27. Wikipedia contributors. Kinect Sports, 2012. http://en.wikipedia.org/wiki/Kinect_Sports.

28. Wikipedia contributors. Street Fighter, 2012. http://en.wikipedia.org/wiki/Street_Fighter.

29. Williams, A., Hughes, L. and Simon, B., Propinquity: exploring embodied gameplay. Ubicomp'10 adjunct papers, ACM, 387-388.

30. Wilson, D. and Sicart, M., Now it's personal: on abusive game design. Conference on the Future of Game Design and Technology, 2010, ACM, 40-47. 